\title{
Lipoxygenases at the heart of atherosclerosis susceptibility
}

\author{
European Journal of Human Genetics (2004) 12, 601-602. doi:10.1038/sj.ejhg.5201231
}

It is well established that high levels of low-density lipoproteins (LDL) play a causal role in the etiology of atherosclerosis and the subsequent development of coronary artery disease (CAD) in man. Mainly through the work in cellular and animal models, a detailed molecular mechanism is emerging, which explains how pathogenic atherosclerotic lesions develop and can lead to myocardial infarction (MI) and stroke. In the prevailing model, LDL becomes oxidatively modified in the vessel wall, initiating the formation of an early fatty streak lesion consisting mainly of macrophages. Subsequently, a series of lipidmodifying enzymes have been identified in the vessel wall, capable of generating inflammatory mediators, which can further stimulate plaque growth by enhancing cellular influx. ${ }^{1}$

As long as 15 years ago, the presence of 12-lipoxygenase was demonstrated in human atherosclerotic plaques, and it was hypothesized that the activity of 12-lipoxygenase was crucial for the development of atherosclerotic disease. ${ }^{2}$ Until recently, however, little evidence was obtained in human studies that supported this mechanism, and current therapies have remained focusing on lipid lowering as the principle therapeutic target. This situation is now likely to change very rapidly due to a series of breakthroughs in the field of human genetics that begin to close the gap between mouse models of disease and human disease.

How did this excitement take off? One of the lipidmodifying enzymes that have generated a lot of interest recently is 5-lipoxygenase $(5 \mathrm{LO})$. $5 \mathrm{LO}$ is produced by macrophages and is an important enzyme in the conversion of the lipid molecule arachidonic acid into leukotrienes. These leukotrienes are inflammatory mediators involved in monocyte attraction. Hence, 5LO may serve as a gene driving chronic inflammation and thereby the progression of atherosclerotic plaques. In line with this hypothesis, 5LO has recently been shown in genetic and knockout studies in mouse to play a major role in promoting plaque growth. ${ }^{3}$ Based on these findings in mice, polymorphisms in the promoter of 5LO have been studied in patients and were correlated with the progression of lesion formation based on measurements of the thickness of the vessel wall. ${ }^{4}$ These seminal observations place genes interacting with $5 \mathrm{LO}$ in a prime position as candidate genes for CAD. Among those genes is ALOX5AP, encoding 5LO-activating protein (FLAP).

From a clinical point of view, however, an important question remains: can genetic mutations in this pathway affect crucial disease end points, such as MI and stroke? A recent study by Helgadottir et $a l^{5}$ from deCODE genetics elaborated successfully on this clinical question, by performing a linkage study in a large set of Icelandic families. Some 296 families were studied, including 713 patients with MI, generating a highly suggestive linkage peak to a locus on chromosome 13q. Subsequently, this chromosomal region was further investigated by association analysis with 120 microsatellite markers in a study including around 800 cases and controls. This screen resulted in the detection of a haplotype spanning two genes including ALOX5AP. Further studies using SNPs identified with certainty one particular ALOX5AP haplotype as the susceptibility gene in man for MI.

This role of ALOX5AP has also been demonstrated in an Icelandic cohort of patients with stroke and in CAD patients from the UK, although the UK patients differ in their risk haplotype. Hence, this genetic study now implicates a second gene of the lipoxygenase pathway in man to be the cause of cardiovascular disease, despite the fact that no a priori assumptions on candidate genes were made. This strong evidence for the role of the lipoxygenase pathways also renders the remaining genes of the pathway as attractive candidates to be studied genetically. Indeed, some of those genes, such as 12LO and phospholipases, have already been implicated in the progression of atherosclerotic lesions in rodent models. ${ }^{6,7}$

The study by Dwyer et l $^{4}$ has also addressed the crucial issue of gene-environment interactions by studying the effect of diet on 5LO promoter polymorphisms. First, a subgroup of carriers of a particular promoter variant was identified, which showed increased carotid artery intimamedia thickness. Second, it was shown in these allele carriers that dietary arachidonic acid, the substrate for 5LO, increased the production of inflammatory mediators, as compared to subjects that were fed a 'marine' diet with N-3 fatty acids.

In addition to diets, drugs are available that can also modulate the lipoxygenase pathway. Particularly, the role of the 5LO pathway in disease is very well known in the 
field of asthma research. ${ }^{8}$ Therefore, a wide range of drugs that effectively block 5LO or leukotriene receptors is on the market. These drugs could now be of interest to prevent MI and stroke. It is very rewarding that these novel findings regarding the genetic susceptibility of CAD are perfectly in tune with the well worked out model for the etiology of atherosclerosis. The mechanism now established offers excellent opportunities to further study the possibilities for dietary prevention and pharmacological intervention.

\section{Marten Hofker}

Marten Hofker is at the Department of Molecular Genetics, Maastricht University, POB 616, 6200MD Maastricht, The

Netherlands.

E-mail: M.Hofker@gen.unimaas.nl

1 Mehrabian M, Allayee H: 5-lipoxygenase and atherosclerosis. Curr Opin Lipidol 2003; 14: 447-457.
2 Steinberg D, Parthasarathy S, Carew TE, Khoo JC, Witztum JL: Beyond cholesterol. Modifications of low-density lipoprotein that increase its atherogenicity. N Engl J Med 1989; 320: 915 -924.

3 Mehrabian $\mathrm{M}$, Allayee $\mathrm{H}$, Wong $\mathrm{J}$ et al: Identification of 5lipoxygenase as a major gene contributing to atherosclerosis susceptibility in mice. Circ Res 2002; 91: 120-126.

4 Dwyer $\mathrm{JH}$, Allayee $\mathrm{H}$, Dwyer $\mathrm{KM}$ et al: Arachidonate 5lipoxygenase promoter genotype, dietary arachidonic acid, and atherosclerosis. N Engl J Med 2004; 350: 29-37.

5 Helgadottir A, Manolescu A, Thorleifsson G et al: The gene encoding 5-lipoxygenase activating protein confers risk of myocardial infarction and stroke. Nat Genet 2004; 36: 233-239.

6 Cyrus T, Witztum JL, Rader DJ et al: Disruption of the 12/15 lipoxygenase gene diminishes atherosclerosis in apo E-deficient mice. J Clin Invest 1999; 103: 1597-1604.

7 Ivandic B, Castellani LW, Wang XP et al: Role of group II secretory phospholipase A2 in atherosclerosis: 1 . Increased atherogenesis and altered lipoproteins in transgenic mice expressing group IIa phospholipase A2. Arterioscler Thromb Vasc Biol 1999; 19: $1284-1290$.

8 Drazen JM, Yandava CN, Dube L et al: Pharmacogenetic association between ALOX5 promoter genotype and the response to anti-asthma treatment. Nat Genet 1999; 22: $168-170$. 